\title{
Kühlbedingungen beim Inverkehrbringen von frischen Fischereierzeugnissen im Einzelhandel
}

\author{
Dr. G. Hauner \\ Bayerisches Landesamt für Gesundheit und Lebensmittelsicherheit, Dienststelle Oberschleißheim, Veterinärstr. 2, 85764 Oberschleißheim
}

\section{Zusätzliche Informationen/Anlagen}

Urteil des VG Regensburg vom 19.11.2001 (rechtskräftig durch Beschluss des Bayerischen Verwaltungsgerichtshofs vom 15.09.2005)

\section{Zusammenfassung/Erläuterung}

In seinem Urteil vom 19.11.2001 vertritt das Verwaltungsgericht Regensburg die Auffassung, dass die Anordnung der Vollzugsbehörde, dass frische Fischereierzeugnisse auch beim Inverkehrbringen in schmelzendem Eis bzw. bei max. $+2{ }^{\circ} \mathrm{C}$ aufzubewahren sind, nicht zulässig sei. Es führt aus, dass es hierfür nach damaligem Rechtsstand lediglich für das Lagern und Befördern, nicht jedoch für das Inverkehrbringen bzw. Anbieten zum Verkauf eine Rechtsgrundlage gebe. Das Urteil erlangte durch den Beschluss des Bayer. Verwaltungsgerichtshofs nach
Rücknahme der Berufung durch die zuständige Vollzugsbehörde Rechtskraft. Anlass für die Anordnung der Vollzugsbehörde waren bei einer Probenentnahme festgestellte Produkttemperaturen von $+7,1$ bzw. $+8,3^{\circ} \mathrm{C}$.

Auch die aktuell geltenden Rechtsvorschriften machen ob gewollt oder ungewollt - diesen Unterschied zwischen den Bedingungen einerseits beim Behandeln, andererseits beim Inverkehrbringen von Lebensmitteln.

\section{Beschluss (einstimmig)}

Der Lebensmittelunternehmer ist auf jeder Handelsstufe für die einwandfreie hygienische Beschaffenheit seiner Produkte verantwortlich. Frischfisch muss bei annähernder Schmelzeistemperatur oder in schmelzendem Eis gelagert und angeboten werden. 